\title{
Nonlinear control by coupled oscillator: from nonholonomic systems to quasi-passive dynamic walker
}

\author{
Yasuhiro Sugimoto $^{1 a)}$, Takeshi Kibayashi ${ }^{1}$, Masato Ishikawa $^{1}$, \\ and Koichi Osuka ${ }^{1,2}$ \\ ${ }^{1}$ Dept. of Mechanical Engineering, Osaka University \\ 2-1, Yamadaoka, Suita, Osaka 565-0871, Japan \\ ${ }^{2}$ CREST, JST, 7 Goban-cho, Chiyoda-ku, Tokyo 102-0076, Japan \\ a)yas@mech.eng.osaka-u.ac.jp
}

Received January 24, 2015; Revised May 1, 2015; Published October 1, 2015

\begin{abstract}
In this paper, we propose a novel control method for nonholonomic constrained systems using coupled oscillators composed through the Kuramoto model. The Kuramoto model is a mathematical model used to describe synchronization phenomenona. We focus on the Kuramoto model because it is able to generate stable rhythmic signals that are modulable for engineering, and because it is sufficiently simple for mathematical analysis. The contributions of this paper are twofold. First, we propose the use of a pair of Kuramoto oscillators as dynamic controllers for a nonholonomic Brockett integrator system; next, we define a feedback control scheme by adjusting its angular velocities based on the system state. We then derive stability criteria for the entire feedback system and examine the effectiveness of the system through several numerical simulations. We also show that the proposed idea is applicable to a twowheeled vehicle system, which is locally equivalent to the Brockett integrator under proper coordinate and input transformations. Second, we apply the proposed method to control the walking distance of a quadrupedal quasi-passive dynamic walker and examine the effectiveness of the proposed method through walking experiments.
\end{abstract}

Key Words: nonholonomic constrained system, kuramoto oscillator model, Brockett integrator, two-wheeled vehicle, quasi-passive dynamic walking

\section{Introduction}

In the field of nonlinear control theory, nonholonomic constrained systems have been extensively investigated since the early $90 \mathrm{~s}$ [1]. Nonholonomic systems are literally defined as a class of mechanical that undergo nonintegrable mechanical constraints. From the viewpoint of nonlinear control, one outstanding feature is that typical nonholonomic systems are not controllable in the linear sense (i.e., corresponding linear approximations fail to be controllable), but they can be controllable in a nonlinear sense by considering the effects of Lie brackets on the input vector fields. Such systems 


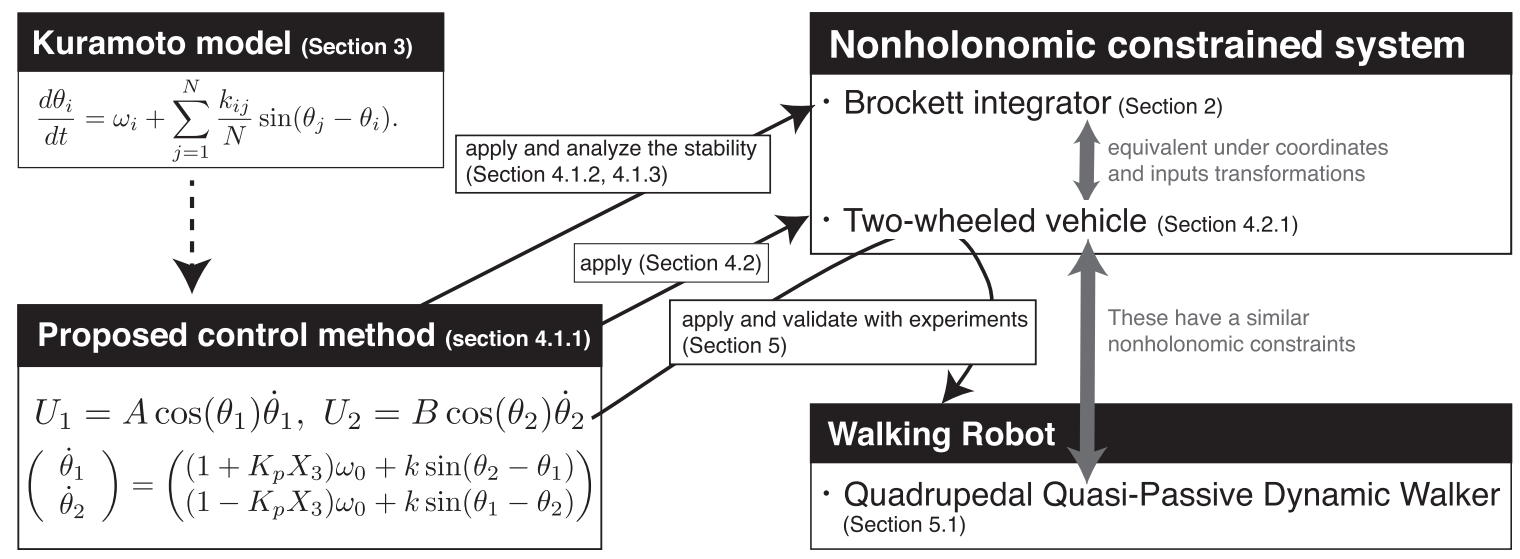

Fig. 1. Relationship among Kuramoto oscillator model, Brockett integrator, Two-wheeled vehicle, Quadrupedal Quasi-Passive Dynamic Walker and the proposed control method in this paper.

often appear in locomotion control problems of mobile or space robots.

A key control strategy for nonholonomic systems involves the use of periodic control inputs with proper phase gaps, such as sine-cosine pairs, because the gaps are approximately proportional to the Lie bracket effects. In this case, the problem is how to create periodic inputs with a proper phase gap. In our previous work [2], a Matsuoka model, which is a mathematical model of neural elements proposed by Matsuoka [3], was used to generate such periodic control inputs. The proposed control strategy was applied to a rolling sphere control problem, and its effect was verified with numerical simulations. However, a sufficient mathematical stability analysis of the proposed method has not yet been conducted.

In this paper, we propose a novel control method for nonholonomic constrained systems based on the Kuramoto model, which is a mathematical model used to describe synchronization phenomena [46]. We focus on the Kuramoto model because it is able to generate stable rhythmic signals that are modulable through engineering, and because it is sufficiently simple for mathematical analysis.

Our primary contribution is roughly composed of two parts. First, we propose the use of a pair of Kuramoto oscillators as dynamic controllers for a nonholonomic Brockett integrator system, and then define a feedback control scheme by adjusting its angular velocities based on the system state. We then derive stability criteria for the entire feedback system and examine its effectiveness through several numerical simulations. We also show that the proposed idea is applicable to a two-wheeled vehicle system, which is locally equivalent to a Brockett integrator under proper coordinates and input transformations. Next, we apply the proposed method to a completely different target: a quadrupedal quasi-passive dynamic walking robot. This walking robot, which we called "Duke," was developed in our previous study [7]. In fact, we revealed that Duke's walking dynamics reflect nonholonomic behavior due to the rolling contact constraints between its feet and the ground, and that Duke is partially similar to a two-wheeled vehicle. This result motivated us to connect these two apparently unrelated topics: the Kuramoto model and the walking robot.

This paper is organized as follows (Fig. 1). Section 2 introduces the Brockett integrator and explains its basic features. Section 3 introduces the Kuramoto oscillator model and describes its interesting properties. In Section 4.1, we propose a novel control method using the Kuramoto model for a nonholonomic constraint system, discuss the stability of the proposed control method, and examine its effectiveness with several numerical simulations. In Section 4.2, the proposed control method is applied to a two-wheeled vehicle system. Section 5 demonstrates the application of the proposed control method to a quadrupedal quasi-passive-dynamic-walking robot and shows the experimental results. Conclusions and future works are discussed in Section 6. 


\section{Brockett integrator}

First, we focus on the so-called Brockett integrator system [8] in particular, which is the simplest example of a nonholonomic system. The state equation of the Brockett integrator is shown as follows:

$$
\begin{aligned}
\left(\begin{array}{c}
\dot{X}_{1} \\
\dot{X}_{2} \\
\dot{X}_{3}
\end{array}\right) & =\left(\begin{array}{cc}
1 & 0 \\
0 & 1 \\
X_{2} & -X_{1}
\end{array}\right)\left(\begin{array}{c}
U_{1} \\
U_{2}
\end{array}\right) \\
& :=g_{\mathbf{1}}(\boldsymbol{X}) U_{1}+\boldsymbol{g}_{\mathbf{2}}(\boldsymbol{X}) U_{2}
\end{aligned}
$$

where $\boldsymbol{X}=\left(X_{1}, X_{2}, X_{3}\right)^{T} \in \mathbb{R}^{3}$ is the system state and $\boldsymbol{U}=\left(U_{1}, U_{2}\right)^{T} \in \mathbb{R}^{2}$ is the control input; $\boldsymbol{g}_{1}$ and $\boldsymbol{g}_{2}$ are the input vector-fields corresponding to the inputs $U_{1}$ and $U_{2}$ respectively. It is clear that $X_{1}$ and $X_{2}$ can be controlled directly by $U_{1}$ and $U_{2}$, whereas $X_{3}$ should be controlled indirectly by appropriate coordination of $U_{1}(t)$ and $U_{2}(t)$, considering the time profiles. For instance, it was shown that periodic inputs $U_{1}$ and $U_{2}$ with proper phase gaps, such as sine-cosine pairs of functions [1] were effective to generate net change for $X_{3}$.

Here, let us define a nonassociative, antisymmetric product between two smooth vector-fields, $\boldsymbol{g}_{\boldsymbol{i}}$ and $\boldsymbol{g}_{\boldsymbol{j}}$, called Lie bracket:

$$
\left[\boldsymbol{g}_{i}, \boldsymbol{g}_{j}\right]=\frac{\partial \boldsymbol{g}_{j}}{\partial \boldsymbol{X}} \boldsymbol{g}_{i}-\frac{\partial \boldsymbol{g}_{i}}{\partial \boldsymbol{X}} \boldsymbol{g}_{j}
$$

Lie bracket plays a significant role in controlling nonlinear systems of the form (2), since it gives us an approximate change of the state vector $X$ under periodic control inputs. Suppose $U_{1}(t)$ and $U_{2}(t)$ are functions of $t$ with period $T$ and zero-average (thus $\int U_{1}(t) d t$ and $\int U_{2}(t)$ are also $T$-periodic). The change of $X$ after one cycle of periodic control, say $X(T)-X(0)$, is approximately parallel to the vector $\left[g_{1}, g_{2}\right]$. Moreover, the amount of this displacement is proportional to the area enclosed by the curve $\left(\int U_{1}(t) d t, \int U_{2}(t) d t\right)$. This is derived from a differential-geometric principle, called area formula $[1,9]$, which is essentially a generalization of the Stokes' theorem.

For example, the Lie bracket of $\boldsymbol{g}_{\mathbf{1}}$ and $\boldsymbol{g}_{\mathbf{2}}$ in the system (1) can be calculated as follows:

$$
\left[\boldsymbol{g}_{1}, \boldsymbol{g}_{2}\right]=[0,0,-2]^{T} \text {. }
$$

The third element of this Lie bracket is nonzero, thus, system (1) can also be maneuvered in the $X_{3}$ direction.

From these facts, it is be expected that $X_{3}$ can be controlled with certain periodic $U_{1}$ and $U_{2}$. In this case, the problem is how to create periodic inputs with a proper phase gap. This is because the area enclosed by the curve of input integrations strongly depends on a phase gap between $U_{1}$ and $U_{2}$. Actually, when $u_{1}=A \sin t$ and $u_{2}=A \sin (t-\psi)$ are used as periodic inputs, the enclosed area $S$, by the curve of input integrations becomes

$$
S=-A^{2} \sin \psi .
$$

In the next section, we propose the use of a couple of Kuramoto oscillators to produce appropriate phase gaps by modulating their parameters.

Remark 1. The Brockett integrator (1) is known as a canonical representation of nilpotent nonholonomic systems. Other equivalent representations include chained systems [10] and power forms [11]. This class of systems can be generalized as "first-order systems [12]", where $\boldsymbol{g}_{\mathbf{1}}(X), \boldsymbol{g}_{\mathbf{2}}(X)$ and $\left[\boldsymbol{g}_{\mathbf{1}}, \boldsymbol{g}_{\mathbf{2}}\right](X)$ are linearly independent to each other at any $X$. Since the proposed method only based on the aforementioned area formula, the method would be applied to the first-order systems with slight customization.

\section{Kuramoto oscillator model}

It is well known that there are many types of synchronization phenomena in autonomous systems, such as mechanical systems, chemical reactions, and circadian rhythms. To describe such synchronization, 


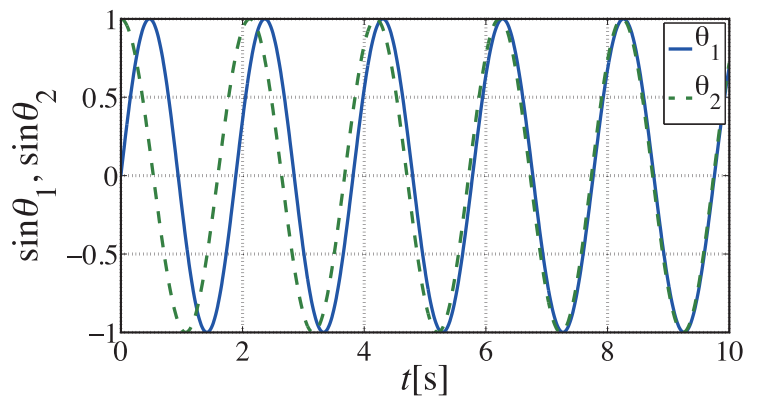

(a) $\omega_{1}=\omega_{2}=\pi$

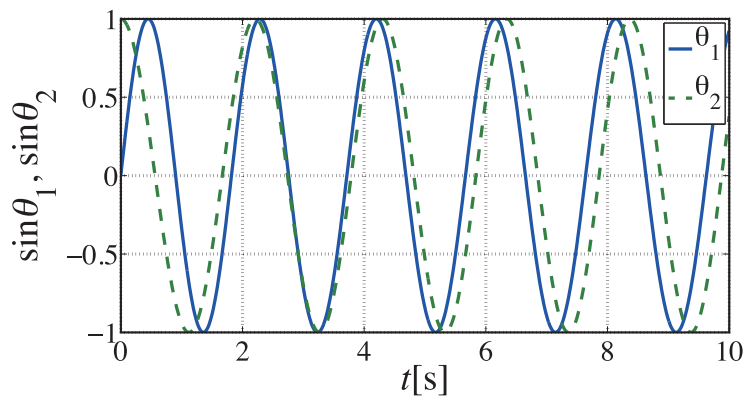

(b) $\omega_{1}=1.05 \pi, \omega_{2}=0.95 \pi$

Fig. 2. Simulation results of Kuramoto oscillator model.

various models have been proposed. The Kuramoto model, first proposed by Kuramoto [4], is a mathematical model used to describe synchronization $[4,5]$. The Kuramoto model, which consists of a population of $N$ coupled phase oscillators $\theta_{i}$ having natural frequencies $\omega_{i}$ and coupling strength $k_{i j}$, is described as

$$
\frac{d \theta_{i}}{d t}=\omega_{i}+\sum_{j=1}^{N} \frac{k_{i j}}{N} \sin \left(\theta_{j}-\theta_{i}\right) .
$$

This model is simple enough to be mathematically tractable, yet sufficiently complex to be nontrivial. For example, when $k_{i j}$ is sufficiently weak, the oscillators run incoherently, whereas beyond a certain threshold, collective synchronization spontaneously emerges. Acebron el at. studied many different models, such as nearest-neighbor coupling, hierarchical coupling, random long-range coupling, or even state-dependent interactions [6]. Moreover, the behavior of each oscillator varies depending on $\omega_{i}$. Figure 2 shows numerical simulation results of the Kuramoto model, which consists of two oscillators $\theta_{1}, \theta_{2}$ :

$$
\frac{d \theta_{1}}{d t}=\omega_{1}+k_{12} \sin \left(\theta_{2}-\theta_{1}\right), \quad \frac{d \theta_{2}}{d t}=\omega_{2}+k_{21} \sin \left(\theta_{1}-\theta_{2}\right) .
$$

Figures 2(a) and (b) show simulation results for when natural frequencies were set as $\omega_{1}=\omega_{2}=\pi$ and $\omega_{1}=1.05 \pi, \omega_{2}=0.95 \pi$, respectively. Other parameters were $k_{12}=k_{21}=1$ and initial conditions were $\theta_{1}(0)=1$ and $\theta_{2}(0)=\pi$. The two oscillators in Fig. 2(a) are synchronized. Indeed $\sin \theta_{1}-\sin \theta_{2}$ converges to zero since $\frac{d}{d t}\left(\theta_{1}-\theta_{2}\right)=-2 \sin \left(\theta_{1}-\theta_{2}\right)$. On the other hand, those in Fig. 2(b) are decoupled. This means that a phase gap between two oscillators can be produced through even a small difference in $\omega_{i}$.

Thus, in this study, we use this dependency on the natural frequency of the Kuramoto model to produce periodic control inputs for the Brockett integrator.

\section{Feedback control for nonholonomic systems using the Kuramoto model}

\subsection{Control of Brockett integrator}

In this section, we propose a novel control method for nonholonomic constrained systems using coupled oscillators composed by using the Kuramoto model. Next, we derive the stability criteria of the Brockett integrator with the proposed method. Finally, we examine the effectiveness of the proposed method through several numerical simulations

\subsubsection{Proposed control method}

Based on Kuramoto model (7), let us consider the following oscillator model with a feedback term,

$$
\left(\begin{array}{c}
\dot{\theta}_{1} \\
\dot{\theta}_{2}
\end{array}\right)=\left(\begin{array}{c}
\left(1+K_{p} X_{3}\right) \omega_{0}+k \sin \left(\theta_{2}-\theta_{1}\right) \\
\left(1-K_{p} X_{3}\right) \omega_{0}+k \sin \left(\theta_{1}-\theta_{2}\right)
\end{array}\right),
$$


where $\omega_{0}$ is the common natural frequency, $k$ is the bonding strength of oscillators, and $K_{p}$ is the feedback gain. As shown, oscillator model (8) includes state $X_{3}$, which modifies the frequency of each oscillator. Consequently, it is expected that these oscillators will show various synchronization patterns depending on state $\boldsymbol{X}$.

Using these oscillators, $\theta_{1}$ and $\theta_{2}$, we propose to assign the following $U_{1}$ and $U_{2}$ as the control inputs for Brockett integrator (1),

$$
U_{1}=A \cos \left(\theta_{1}\right) \dot{\theta}_{1}, \quad U_{2}=B \cos \left(\theta_{2}\right) \dot{\theta}_{2},
$$

where $\int U_{1} d t=A \sin \theta_{1}$ and $\int U_{2} d t=B \sin \theta_{2}$.

\subsubsection{Stability analysis}

In this section, we show that the proposed control inputs (9) and oscillator model (8) can deliver $X_{3}$ in the system (1) to 0 if the phase difference between the oscillators (8) is sufficiently small, that is, $\sin \left(\theta_{1}-\theta_{2}\right) \approx \theta_{1}-\theta_{2}$. If the target state $X_{3}^{*}$ is not 0 , an coordinate transformation $\tilde{X}_{3}=X_{3}-X_{3}^{*}$ enables $X_{3}$ to converge to $X_{3}^{*}$.

Let $\Theta$ and $\dot{\Theta}$ be $\Theta:=\theta_{1}-\theta_{2}$ and $\dot{\Theta}:=d \Theta / d t$. The Brockett integrator system (1) with inputs (9) can be rewritten to

$$
\begin{aligned}
\dot{X}_{3} & =-A B \omega_{0} \sin \Theta+A B \sin \left(2 \omega_{0} t\right)\left(K_{p} \omega_{0} X_{3}-k \sin \Theta\right) \\
\dot{\Theta} & =2\left(K_{p} \omega_{0} X_{3}-k \sin \Theta\right) .
\end{aligned}
$$

When $\sin \Theta \approx \Theta$, these equations can be linearized to

$$
\begin{aligned}
\dot{X}_{3} & =-A B \omega_{0} \Theta+A B \sin \left(2 \omega_{0} t\right)\left(K_{p} \omega_{0} X_{3}-k \Theta\right) \\
\dot{\Theta} & =2\left(K_{p} \omega_{0} X_{3}-k \Theta\right) .
\end{aligned}
$$

The stability condition of the linearized system (10) and (11) is given by the following theorem.

Theorem 1. The origin $\left(X_{3}, \Theta\right)=(0,0)$ of (10), (11) is asymptotically stable if $k>A B K_{p} \omega_{0} / 2$.

Proof. At first, we introduce Krasovski theorem [13], which gives a sufficient condition for a stability of periodic dynamical system.

Krasovski theorem. Let $\dot{\boldsymbol{x}}=\boldsymbol{f}(t, \boldsymbol{x})$ be a differential equation which is periodic in $t$ with period $T$. Suppose there exists a $\mathbb{C}^{1}$ function $V: I \times \Omega \rightarrow \mathbb{R}$, periodic in $t$ with period $T$, such that for some function $a \in \mathbb{R}$ and every $(t, x) \in I \times \Omega$ :

1) $V(t, \boldsymbol{x}) \geq a(\|\boldsymbol{x}\|) ; V(t, 0)=0$;

2) $\dot{V}(t, \boldsymbol{x}) \leq 0$;

3) except for the origin, $M$ contains no complete positive semi-trajectory where $M=\{(t, \boldsymbol{x}) \in$ $I \times \Omega: \dot{V}=0\}$

are satisfied.

Then, the origin is uniformly asymptotically stable.

Using this theorem, we show the stability of the system (10), (11) .

Let $\boldsymbol{x}=\left(X_{3}, \Theta\right)$. Here, $V(t, \boldsymbol{x})$ and $a(\|\boldsymbol{x}\|)$ are defined as follows,

$$
\begin{aligned}
V(t, \boldsymbol{x}) & =2\left(K_{p} \omega_{0} X_{3}-k \Theta\right)^{2}+A B K_{p} \omega_{0}^{2} \Theta^{2} \\
& =: \alpha X_{3}^{2}+2 \beta X_{3} \Theta+\gamma \Theta^{2} \\
a(\|\boldsymbol{x}\|) & =\frac{\alpha+\gamma+\sqrt{(\alpha-\gamma)^{2}+4 \beta^{2}}}{2}\left(X_{3}^{2}+\Theta^{2}\right) .
\end{aligned}
$$

It is obvious that $V(t, \mathbf{0})=0$. From Eqs. (13) and (14), $V(t, \boldsymbol{x})-a(\|\boldsymbol{x}\|)$ yields 


$$
\begin{aligned}
& V(t, \boldsymbol{x})-a(\|\boldsymbol{x}\|) \\
&= \frac{\alpha-\gamma+\sqrt{(\alpha-\gamma)^{2}+4 \beta^{2}}}{2} X_{3}^{2}+2 \beta X_{3} \Theta+\frac{-\alpha+\gamma+\sqrt{(\alpha-\gamma)^{2}+4 \beta^{2}}}{2} \\
& \Theta^{2} \\
&=\left(\sqrt{\frac{\alpha-\gamma+\sqrt{(\alpha-\gamma)^{2}+4 \beta^{2}}}{2}} X_{3}+\sqrt{\frac{-\alpha+\gamma+\sqrt{(\alpha-\gamma)^{2}+4 \beta^{2}}}{2}} \Theta\right)^{2} \\
& \geq 0 .
\end{aligned}
$$

From the inequality, it is verified that the condition 1) in Krasovski Theorem is satisfied. Next, differentiating $V(t, \boldsymbol{x})$ with respect to $t, \dot{V}(t, \boldsymbol{x})$ can be derived as

$$
\begin{aligned}
\dot{V} & =4\left(K_{p} \omega_{0} X_{3}-k \Theta\right)\left(K_{p} \omega_{0} \dot{X}_{3}-k \dot{\Theta}\right)+2 A B K_{p} \omega_{0}^{2} \dot{\Theta} \Theta \\
& =2 \dot{\Theta}\left\{K_{p} \omega_{0}\left(-A B \omega_{0} \Theta+A B \sin \left(2 \omega_{0} t\right) \dot{\Theta} / 2\right)-k \dot{\Theta}\right\}+2 A B K_{p} \omega_{0}^{2} \dot{\Theta} \Theta \\
& =\left(A B K_{p} \omega_{0} \sin \left(2 \omega_{0} t\right)-2 k\right) \dot{\Theta}^{2} \\
& \leq\left(A B K_{p} \omega_{0}-2 k\right) \dot{\Theta}^{2} .
\end{aligned}
$$

From Eq. (17), it can be said that the condition 2) in Krasovski Theorem is satisfied if $k>A B K_{p} \omega_{0} / 2$.

Finally, from Eqs. (16), (11), $M$ can be described as:

$$
M=\left\{\left(t, X_{3}, \Theta\right): K_{p} \omega_{0} X_{3}-k \Theta=0\right\}
$$

Suppose a $\left(t^{*}, X_{3}^{*}, \Theta^{*}\right) \in M$. If a trajectory which starts from $\left(t^{*}, X_{3}^{*}, \Theta^{*}\right)$ are always contained in $M$, the equation $K_{p} \omega_{0} \dot{X}_{3}-k \dot{\Theta}=0$ has to be satisfied at least. From Eqs. (10), (11), the time evolution of $\left(X_{3}, \Theta\right)$ from $\left(t^{*}, X_{3}^{*}, \Theta^{*}\right)$ can be derived as:

$$
\dot{X}_{3}=\omega_{0} \Theta^{*}, \quad \dot{\Theta}=0 .
$$

Therefore, only the origin can stay on $M$, and then, it is verified that the condition 3) in Krasovski Theorem is satisfied.

The above discussion provides that all conditions of Krasovski Theorem are satisfied if $k>$ $A B K_{p} \omega_{0} / 2$. Therefore, the origin $\boldsymbol{x}=\left(X_{3}, \Theta\right)=(0,0)$ of the system (10), (11) is asymptotically stable if $k>A B K_{p} \omega_{0} / 2$.

From the theorem, it can be said that the proposed control method can deliver $X_{3}$ to 0 if the phase difference between the oscillators is sufficiently small. To prevent that the phase difference between the oscillators $\theta_{1}-\theta_{2}$ becomes large, it is necessary that the initial phase difference $\theta_{1}(0)-$ $\theta_{2}(0)$ is small. The initial phases $\theta_{1}(0)$ and $\theta_{2}(0)$ are calculated from $\theta_{1}(0)=\arcsin \left(X_{1}(0) / A\right)$ and $\theta_{2}(0)=\arcsin \left(X_{2}(0) / B\right)$ based on relationships $X_{1}=\int U_{1} d t=A \sin \theta_{1}$ and $X_{2}=\int U_{2} d t=B \sin \theta_{2}$. Consequently, it is necessary that the initial state difference $X_{1}(0)-X_{2}(0)$ is sufficiently small for $X_{3}$ to be converged to 0 with the proposed control method.

\subsubsection{Simulation result}

We verified the proposed control method through numerical simulations. Figure 3 shows the simulation results when the initial state was $\boldsymbol{X}=[0,0.5,5]^{T}$ and the feedback gain was $K_{p}=0.1$ in Eq. (8). Other parameters were set as $k=\pi, \omega_{0}=\pi$ and $A=B=1$, such that the condition $k>A B K_{p} \omega_{0} / 2$ was satisfied.

Figure 3(a) shows the trajectories of $X_{1}, X_{2}$, and $X_{3}$ and Fig. 3(b) shows the phase difference $\theta_{1}-\theta_{2}$. As shown, $X_{3}$ successfully converged to 0 and phase difference $\theta_{1}-\theta_{2}$ also converged to 0 . Therefore, it was verified that $X_{3}$ in Brockett integrator (1), which corresponds to the direction of the Lie bracket, can be controlled by proposed control method (8). Figure 4 shows another simulation result for when the feedback gain was changed to $K_{p}=0.3$. In this case, the phase difference converged to $2 \pi$, whereas $X_{3}$ also successfully converged to 0 . Figure 5 shows the simulation results for when $K_{p}=0.1$ and $k=0.01 \pi$, which does not satisfy the condition $k>A B K_{p} \omega_{0} / 2$. In this case, $\theta_{1}$ and 


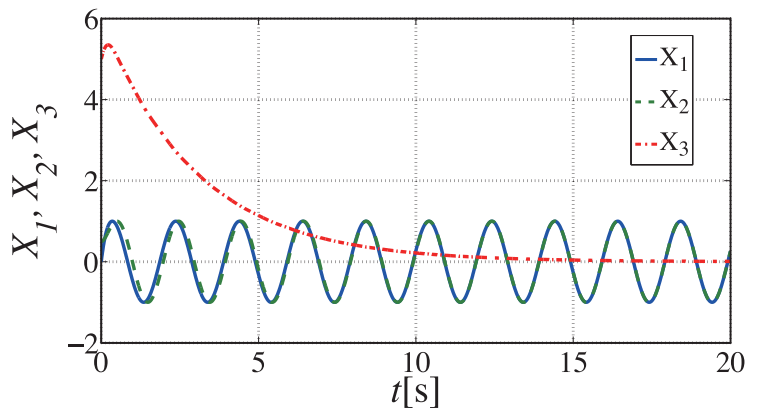

(a) $X_{1}, X_{2}, X_{3}$

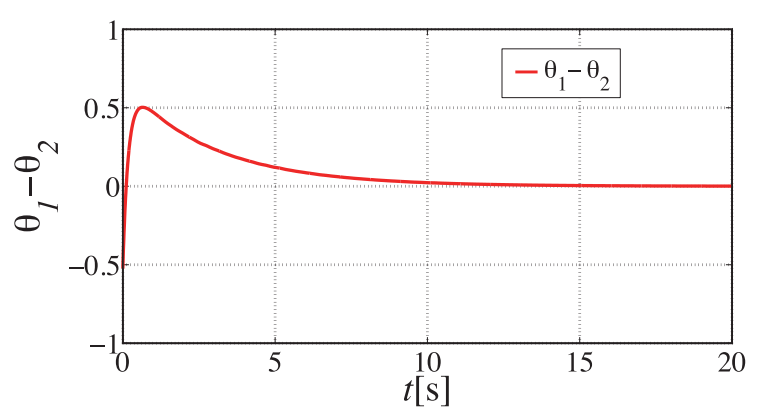

(b) Phase difference $\theta_{1}-\theta_{2}$

Fig. 3. Control of Brockett integrator with proposed method $\left(K_{p}=0.1\right.$, $k=\pi)$.

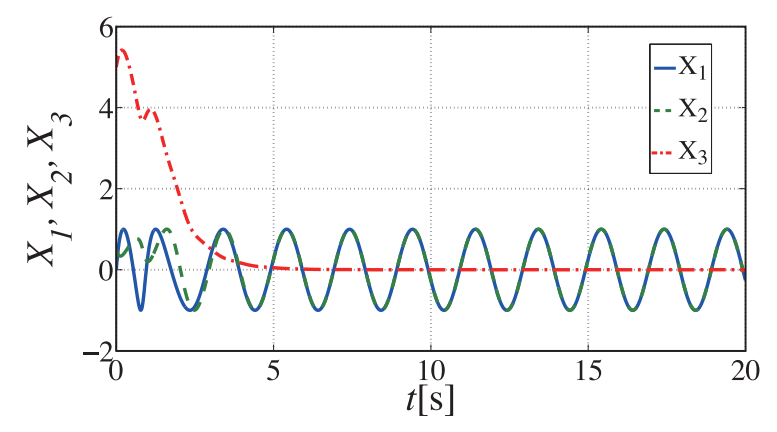

(a) $X_{1}, X_{2}, X_{3}$

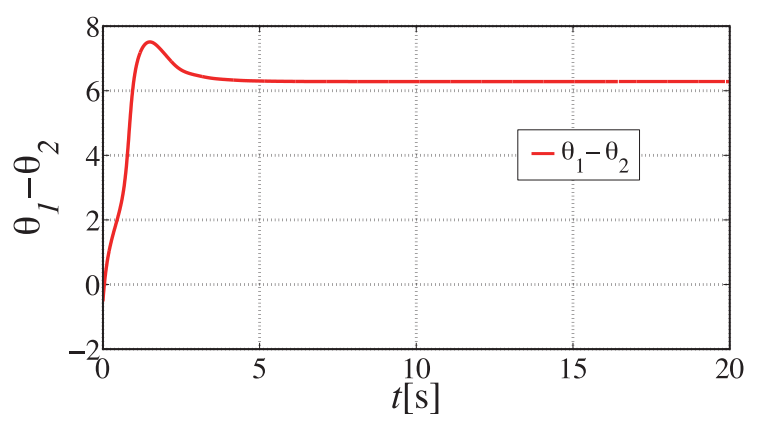

(b) Phase difference $\theta_{1}-\theta_{2}$

Fig. 4. Control of Brockett integrator with proposed method $\left(K_{p}=0.3\right.$, $k=\pi)$.

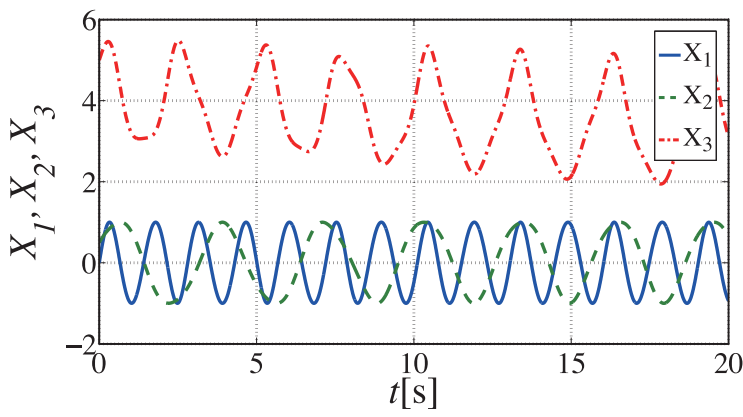

(a) $X_{1}, X_{2}, X_{3}$

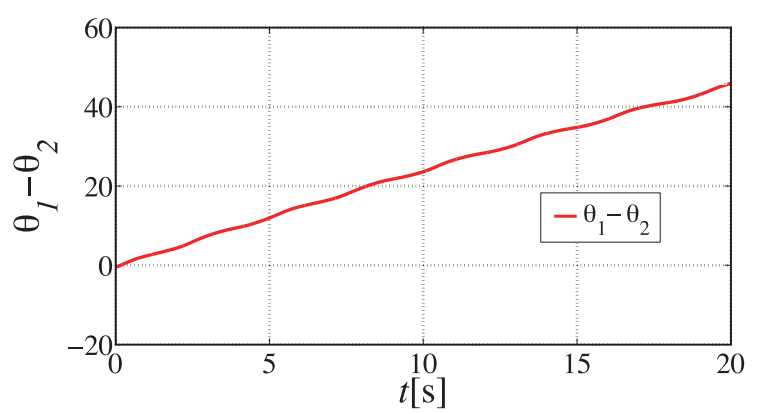

(b) Phase difference $\theta_{1}-\theta_{2}$

Fig. 5. Control of Brockett integrator with proposed method $\left(K_{p}=0.1\right.$, $k=0.01 \pi)$.

$\theta_{2}$ are not synchronized and $X_{3}$ does not converge. These results verify that the proposed method under the condition $k>A B K_{p} \omega_{0} / 2$ can control the Brockett integrator system. Here, it should be noted that 1) the condition $k>A B K_{p} \omega_{0} / 2$ is only a sufficient condition, that is, $X_{3}$ may converge to 0 even with $k \leq A B K_{p} \omega_{0} / 2$ depending on a initial state, 2) the choice of $k$ and $K_{p}$ affects the convergence property as shown in Figs. 3,4 and 5. The dailed analysis of the convergence property depending on $k$ and $K_{p}$ is left as a future work.

\subsection{Control of two-wheeled vehicle}

In this section, we apply the proposed approach to the control of a two-wheeled vehicle, which is one of the nonholonomic systems as well as Brockett integrator. 


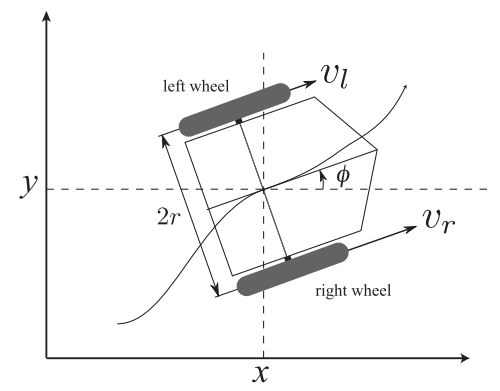

Fig. 6. Kinematic model of two-wheeled vehicle.

\subsubsection{Transformation from Brockett integrator to two-wheeled vehicle}

Figure 6 shows a kinematic model of the two-wheeled vehicle. Under nonslip and nonslide assumptions, the kinematic equation of the two-wheeled vehicle model is shown as follows:

$$
\left(\begin{array}{c}
\dot{x} \\
\dot{y} \\
\dot{\phi}
\end{array}\right)=\left(\begin{array}{cc}
\cos \phi / 2 & \cos \phi / 2 \\
\sin \phi / 2 & \sin \phi / 2 \\
1 / 2 r & -1 / 2 r
\end{array}\right)\left(\begin{array}{c}
v_{r} \\
v_{l}
\end{array}\right),
$$

where the position $(x, y)$ is the center of gravity of vehicle; $v_{r}$ and $v_{l}$ are velocities of the right and left wheels, respectively; $\phi$ is the attitude of the vehicle; and $2 r$ is the vehicle width.

In nonlinear control theory, it is known that a two-wheeled vehicle system is classified into the same class of nonholonomic systems as the Brockett integrator and that these two systems are equivalent under coordinates and input transformations as long as $|\phi| \leq \pi / 2[10,11]$. Such transformation between Brockett integrator (1) and two-wheeled vehicle model (20) can be derived as follows:

$$
X_{1}=x, X_{2}=\tan \phi, X_{3}=2 y-x \tan \phi
$$

and

$$
\begin{aligned}
& v_{r}=\frac{U_{1}}{\cos \left(\tan ^{-1} X_{2}\right)}+r \cos ^{2}\left(\tan ^{-1} X_{2}\right) U_{2}, \\
& v_{l}=\frac{U_{1}}{\cos \left(\tan ^{-1} X_{2}\right)}-r \cos ^{2}\left(\tan ^{-1} X_{2}\right) U_{2} .
\end{aligned}
$$

Under the non-slip and non-slide constraints, the two-wheeled vehicle can not move toward the positive direction of y-axis, which is vertical to the wheels, in a moment. Then, in order to control $y$ of the two-wheeled vehicle (20), we try to control $X_{3}$ in Eq. (21) with the proposed control method (8) and (9).

Then, we focus on the equivalence between Brockett integrator and the two-wheeled vehicle system. To control $y$ of the two-wheeled vehicle (20), we attempt to control $X_{3}$ in Eq. (21) by using proposed control methods (8) and (9), which were used to control $X_{3}$ of Brockett integrator."

\subsubsection{Simulation result}

We confirmed the effects of the proposed method through numerical simulation. The physical parameter was set as $2 r=0.24$. The control parameters in Eq. (8) were set as $k=\pi, \omega_{0}=\pi, K_{p}=0.3$ and $A=B=1$. Figure 7 shows the simulation results with the initial state $(x, y, \phi)^{T}=(0,0,-3)^{T}$. Figures $7(\mathrm{a})$ and $7(\mathrm{~b})$ show the trajectories of $(x, y, \phi)$ and $X_{3}=2 y-x \tan \phi$, respectively. Although $y$ continued to oscillate slightly, the trajectory of $y$ successfully converged to near 0 and $X_{3}=2 y-x \tan \phi$ converged to 0 . From this result, it is verified that the proposed control method (8) can be also applied to the control of $y$ in the two-wheeled vehicle, which corresponds to the vertical direction of the wheels.

\section{Feedback control of quadrupedal quasi-passive dynamic walking robot}

Finally, we apply the proposed control method to controlling the quadrupedal quasi-passive dynamic walking robot and verify its effectiveness through experiments with our quadrupedal walking robot. 


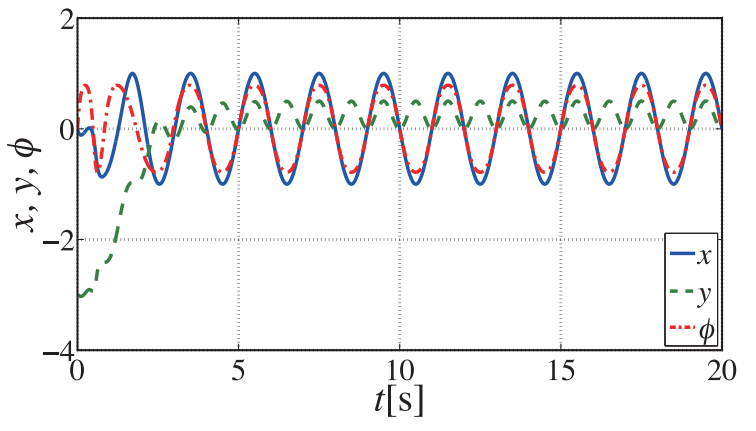

(a) state of two-wheeled vehicle $(x, y, \phi)$

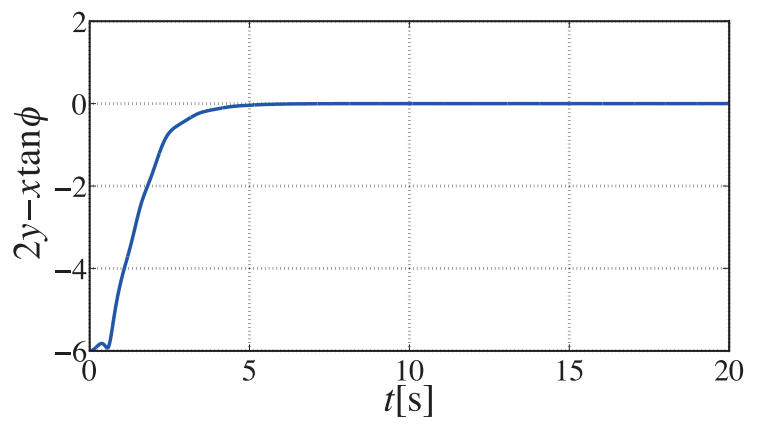

(b) $X_{3}=2 y-x \tan \phi$

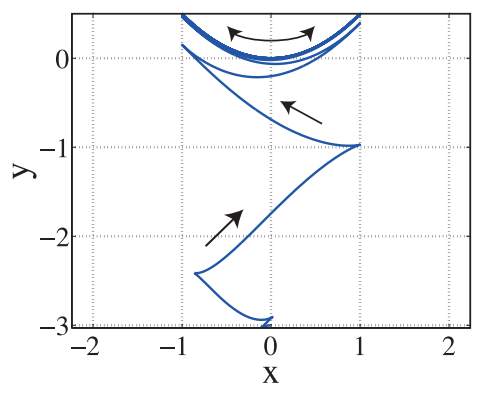

(c) trajectory of $(x, y)$

Fig. 7. Control of two-wheeled vehicle with the proposed control method.

\subsection{Passive dynamic walking}

Passive dynamic walking (PDW) describes a class of mechanical devices that are able to walk down a shallow slope with no actuation $[14,15]$. The PDW robot consists of very simple mechanisms. By using its dynamics and gravity, it can achieve very smooth walking, similar to that of a human, with no actuation or control. It is also well known that PDW robots show chaotic behavior [16-18], such as entertainment and bifurcation phenomena. The entrainment phenomenon of PDW means that even if the robot starts walking under different initial conditions, its walking converges to a specific trajectory. Another interesting type of behavior is bifurcation phenomenon. Depending on changes in slope angle or the robot's parameters, normal (one-period) stable walking bifurcates to two-period walking. Such period doubling, such as bifurcation to four-period from two-period walking or to eight-period from four-period walking, can also occur. These phenomena were verified not only by numerical simulations but also by experiments with real walking robots [18]. Considering such interesting features, much attention has focused on PDW as an approach for producing walking robots $[16,19]$.

Until now, PDW has primarily been studied with bipedal robots. One reason is that PDW appeared to be a breakthrough in the realization of a smooth bipedal walking robot, which has been considered to be quite difficult. However, there are many walking creatures that have more than four legs. To pursue the principle of legged locomotion, it is interesting and critical to analyze whether a PDW robot with four legs or more is achievable. In fact, several studies are related to quadrupedal PDW [20-22]. Our group developed several multi-legged PDW robots named Jenkka I, II, and III. Jenkka-I has four legs, Jenkka-II has 6-legs and Jenkka-III has 20-legs. The leg number of these robots can be reduced because of their special structure. By using these robots, we verified that not only bipedal PDW but also multi-legged (quadruped, hexapod ... 20-legged) PDW is achievable $[22,23]$. With an appropriate initial condition, these robots can excite lateral oscillation on a slope. Depending on its spherical sole shape, each leg can be swung forward in keeping with the excited lateral rocking motion. The lateral oscillation and leg swing motion are constantly repeated, thus these robots achieves locomotion.

\subsection{Quadrupedal quasi-passive dynamic walking Robot "Duke"}

One research topic on PDW is to apply PDW phenomena to the control of a walking robot on level ground. Contrary to walking down a slope, energy input is essential for continuous walking on level ground. Certain researchers have achieved smooth and high-energy efficiency robot walking by using 
the idea of PDW and certain small input torque [19,24-26]. We call this concept "quasi-passive dynamic walking" [26].

Combining the concept of quasi-PDW and quadrupedal-PDW, we developed a quadrupedal quasipassive dynamic walking robot, Duke (Fig. 8) [7]. This robot was designed based on Jenkka II and III and was able to walk only on level ground by exerting little energy in the lateral direction using invert pendulums (Fig. 8(a)). Servo motors are located at the roots of the invert pendulums; the motions of these invert pendulums can be controlled separately by the servo motors. The fundamental structural element is a two-legged PDW walker, and the two elements are connected by a "trunk." The legs of two-legged PDW are linked with passive joints and can swing individually (Fig. 8(b)). The trunk is twistable; that is, the trunk can offer one degree of freedom of relative rotation to the front and rear two-legged elements. Because of this structure, it is feasible to create a phase difference between each two-legged element by using independent inputs to the front and rear servo motors, $\phi_{f}(t), \phi_{h}(t)$ (Fig. 8(b)).

In our previous study, we applied Eqs. (24), (25) as periodic inputs to the front and rear servo motors:

$$
\begin{aligned}
& \phi_{f}(t)=A \sin \left(\frac{2 \pi}{T} t\right), \\
& \phi_{h}(t)=A \sin \left(\frac{2 \pi}{T} t-\psi\right),
\end{aligned}
$$

where $A[\mathrm{rad}], T[\mathrm{~s}]$ and $\psi[\mathrm{rad}]$ are variable parameters referring to amplitude, period, and phase difference, respectively. We verified that Duke can achieve level walking. Additionally, we verified that its walking speed was changed depending on $\psi$, and that the average walking speed was maximum specifically when $\psi=3 \pi / 2[\mathrm{rad}]$. This result suggested a relation between Duke and the nonholonomic constraint system through Eq. (5). As a result of analyses based on the nonlinear control theory, we revealed that Duke had a nonholonomic constraint that corresponds to that of the two-wheeled vehicle and that the motion of the two-wheeled vehicle and Duke could be correlated via

$$
\dot{\phi}_{f}(t)=\frac{1}{r} v_{l}, \quad \dot{\phi}_{h}(t)=\frac{1}{r} v_{r},
$$

where the walking direction of Duke corresponds in the vertical direction to the wheels of the twowheeled vehicle. We concluded that Duke's locomotion consisted of Lie bracket motion derived from a leg swinging motion and a sphere rolling motion in the sole, and that the sole shape contributes to the transitions in walking speed, depending on the phase differences in inputs that exerted the lateral oscillation.

These results suggest the potential to control Duke by proposed control method (9), which can control nonholonomic constraint systems, such as Brockett integrator and two-wheeled vehicles. Next, we apply the proposed control method to controlling the walking distance of Duke.

\subsection{Experimental Result}

We confirmed the effects of the proposed method through walking experiments with the quadrupedal quasi-PDW robot Duke. Let us define the posture angle $\phi$ and position $(x, y)$, which is the center of the robot, as shown in Fig. 8(c). To associate with the result in Section 4.2, Duke's walking direction was coincided with the positive direction of the $y$-axis. Position $(x, y)$ and posture angle $\phi$ were measured with a motion capture system. Inputs to servo motors, $\phi_{f}(t)$ and $\phi_{h}(t)$ were calculated as follows; 1) $\left(X_{1}, X_{2}, X_{3}\right)$ was calculated based on the measured $(x, y, \phi)$ and Eq. $\left.(21), 2\right) U_{1}$ and $U_{2}$ were calculated based on $\left(X_{1}, X_{2}, X_{3}\right)$ and Eqs. (8), (9), 3) $v_{r}$ and $v_{l}$ were calculated based on $U_{1}, U_{2}$ and Eqs. $(22),(23), 4) \dot{\phi}_{f}$ and $\dot{\phi}_{r}$ calculated based on $v_{r}, v_{l}$ and Eq. $\left.(26), 5\right) \phi_{f}$ and $\phi_{h}$ were calculated by numerical integration of $\dot{\phi}_{f}$ and $\dot{\phi}_{r}$. Calculated inputs $\phi_{f}$ and $\phi_{h}$ were wirelessly transmitted to a microcomputer on Duke. The microcomputer drived servo motors based on $\phi_{f}$ and $\phi_{h}$.

Figures 9 and 10 show the experiment results for when the initial state was $(x(0), y(0), \phi(0))^{T}=$ $(0,-0.5,0)^{T}$ and the target state was $y^{*}=0$. The parameters were set as $k=3 \pi, \omega_{0}=2 \pi / 1.5$, 


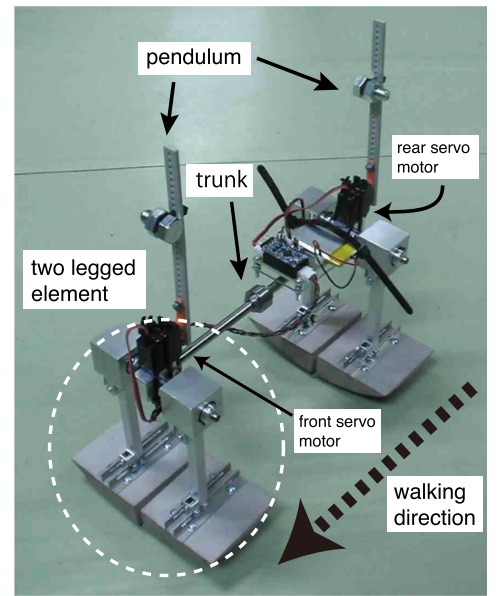

(a) Overview of "Duke"

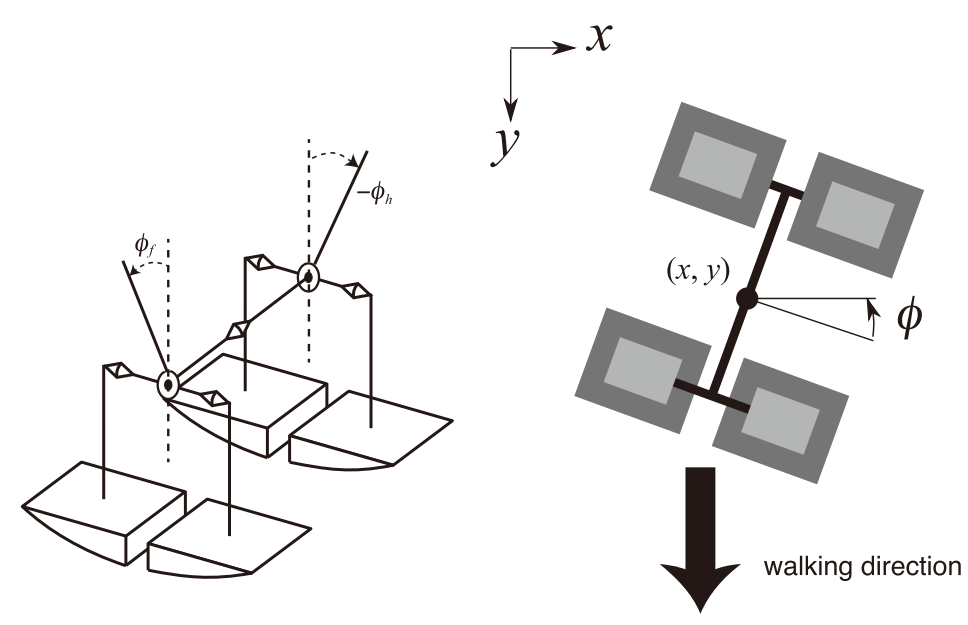

(b) structure of Duke (c) coordinate $(x, y, \phi)$ of Duke

Fig. 8. Quasi-passive dynamic walking robot "Duke".

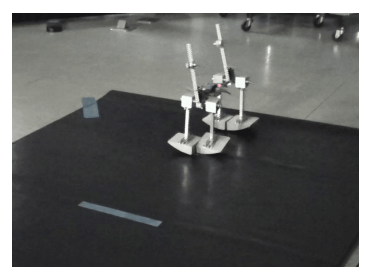

(a) $\mathrm{t}=0$

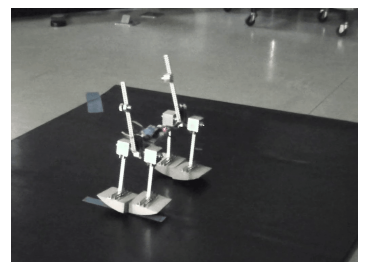

(e) $t=12$

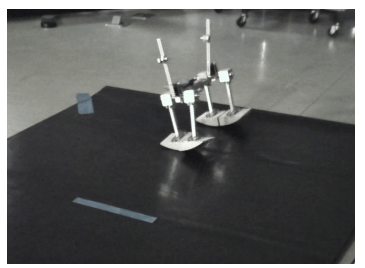

(b) $t=3$

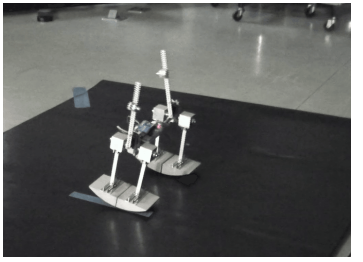

(f) $\mathrm{t}=15$

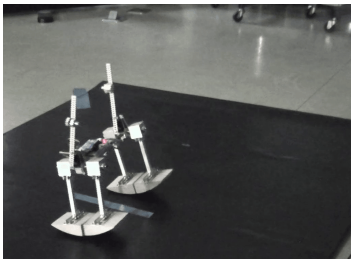

(i) $\mathrm{t}=24$

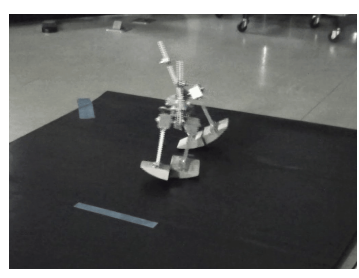

(c) $\mathrm{t}=6$

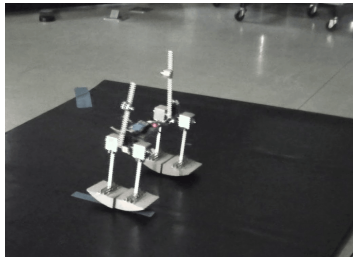

(g) $t=18$

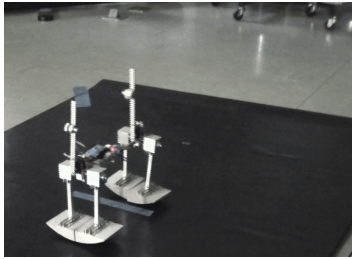

(j) $\mathrm{t}=26$

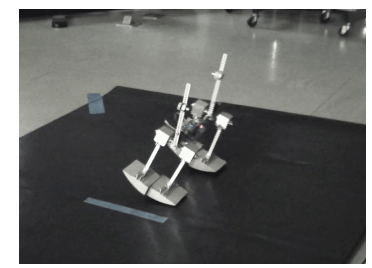

(d) $\mathrm{t}=9$

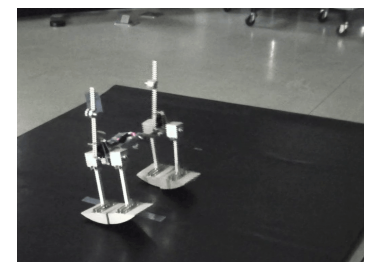

(h) $\mathrm{t}=21$

Fig. 9. Snapshot of walking experiment with Quadrupedal quasi-passive dynamic walking Robot "Duke".

$K_{p}=2, A=0.1$ and $B=0.3$. Figure 9 shows a snapshot of experimental results for Duke with proposed control method. Figures $10(\mathrm{a})$ and $10(\mathrm{~b})$ show walking distance $y$ and trajectory $(x, y)$, respectively. First, Duke walked forward while repeating lateral oscillation of each two-legged element. Gradually, Duke approached $y^{*}$ and the phase difference between lateral oscillation and walking velocity decreased. Finally, Duke began to stamp around $y \approx 0.15[\mathrm{~m}]$.

From the result, the effectiveness of the proposed control method for the control of Duke was confirmed to some extent. However, some steady-state deviation was observed. This is thought to be due to a leg swinging motion, which was not considered in the two-wheeled vehicle model. To reduce the error, it may be effective to add an integral controller into the proposed controller. This issue is left as a future work. 


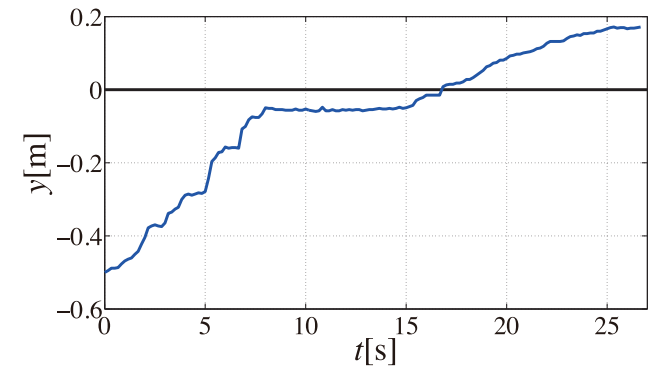

(a) walking distance $y$

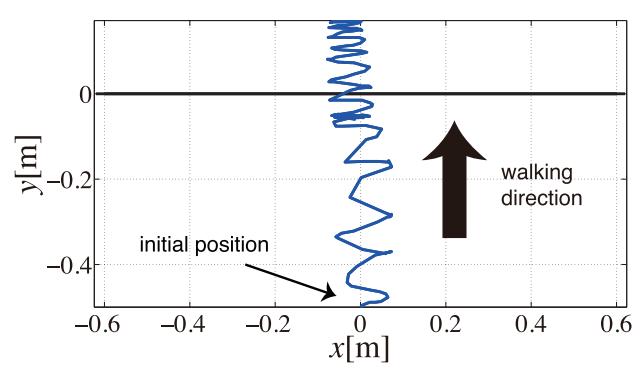

(b) Trajectory of $(x, y)$

Fig. 10. Experimental: feedback control of walking distance.

\section{Conclusion}

In this study, we proposed a feedback control method for nonholonomic constrained systems based on the Kuramoto model and verified the stability of the Brockett integrator system with the proposed control method. Through numerical simulations, we verified that the control method can control the Brockett integrator and two-wheeled vehicle, which are well-known nonholonomic constrained systems. Finally, we applied the proposed method to control the walking distance of our quadrupedal quasiPDW robot, Duke. As described in Section 5.1, there are only several studies related to quadrupedal PDW compared to the research of bipedal PDW. It is interesting and significant that the result of this paper can contributes to not only the research of nonholonomic constrained systems but also the research of quadrupedal-PDW by focusing on the nonholonomic constraint.

The stability analysis in this paper was limited by the assumption that the phase difference is relatively small. To apply this method to more complicated systems, it should be extended in future work.

\section{Acknowledgments}

This work was partially supported by the Grant-in-Aid for Young Scientists (B), No.26870337 from JSPS (the Japan Society for the Promotion of Science) and CREST, JST.

\section{References}

[1] R. Murray and S. Sastry, "Nonholonomic motion planning: steering using sinusoids," IEEE Transactions on Automatic Control, vol. 38, no. 5, pp. 700-716, 1993.

[2] K. Kishimoto, M. Ishikawa, Y. Sugimoto, and K. Osuka, "Feedback control for nonholonomic," Nonlinear Theory and Its Applications, IEICE, vol. 4, no. 4, pp. 365-374, 2013.

[3] K. Matsuoka, "Sustained oscillations generated by mutually inhibiting neurons with adaptation," Biological cybernetics, vol. 52, no. 6, pp. 367-376, 1985.

[4] Y. Kuramoto, "Self-entrainment of a population of coupled non-linear oscillators," in International symposium on mathematical problems in theoretical physics, pp. 420-422, 1975.

[5] Y. Kuramoto, D. Battogtokh, and H. Nakao, "Multiaffine chemical turbulence," Physical review letters, vol. 81, no. 16, pp. 3543-3546, 1998.

[6] J.A. Acebron, L.L. Bonilla, C.J.P. Vicente, F. Ritort, and R. Spigler, "The Kuramoto model : A simple paradigm for synchronization phenomena," Reviews of modern physics, vol. 77, no. 1 , pp. 137-185, 2005.

[7] T. Kibayashi, Y. Sugimoto, M. Ishikawa, K. Osuka, and Y. Sankai, "Experiment and analysis of quadrupedal quasi-passive dynamic walking robot "Duke"," in Proc. of 2012 IEEE/RSJ International Conference on Intelligent Robots and Systems (IROS2012), pp. 299-304, 2012.

[8] R. Brockett, "Asymptotic stability and feedback stabilization," in Differential Geometric Control Theory. Springer-Verlag, vol. 27, pp. 181-191, 1983.

[9] N. Leonard and P. Krishnaprasad, "Motion control of drift-free, left-invariant systems on lie groups," IEEE Trans. on Automatic Control, vol. 40, no. 9, pp. 1539-1554, 1995. 
[10] O. Sordalen and O. Egeland, "Exponential stabilization of nonholonomic chained systems," IEEE Trans. on Automatic Control, vol. 40, no. 1, pp. 35-49, 1995.

[11] C. Samson, "Control of chained systems application to path following and time-varing pointstabilization of mobile robots," IEEE Trans. on Automatic Control, vol. 40, no. 1, pp. 64-77, 1995.

[12] T. Urakubo, K. Tsuchiya, and Y. Tada, "Extended Lyapunov control of first order systems and its applications," in Proc. of the 41st SICE Annual Conference, pp. 2208-2213, 2002.

[13] N. Rouche, P. Habets, and M. Laloy, Stability Theory by Liapunov's Direct Method. SpringerVerlag, 1977.

[14] T. McGeer, "Passive Dynamic Walking," The International Journal of Robotics Research, vol. 9, no. 2, pp. 62-82, 1990.

[15] H. Collins, M. Wisse, and A. Ruina, "A three-dimensional passive-dynamic walking robot with two legs and knees," The International Journal of Robotics Research, vol. 20, no. 7, pp. 607-615, 2001.

[16] A. Goswami, B. Thuilot, and B. Espiau, "A study of the passive gait of a compass-like biped robot: Symmetry and chaos," The International Journal of Robotics Research, vol. 17, no. 12, pp. 1282-1301, 1998.

[17] M. Garcia, A. Chatterjee, and A. Ruina, "Efficiency, speed, and scaling of two-dimensional passive-dynamic walking," Dynamics and Stability of Systems, vol. 15, no. 2, pp. 75-99, June 2000 .

[18] K. Osuka and K. Kirihara, "Motion analysis and experiments of passive walking robot QUARTET II," in Proc. of IEEE ICRA2000, vol. 3, pp. 3052-3056, 2000.

[19] S. Collins, A. Ruina, R. Tedrake, and M. Wisse, "Efficient bipedal robots based on passive dynamic walkers," Science Magazine, vol. 307, no. 5712, pp. 1082-1085, 2005.

[20] A. Smith and M. Berkemeier, "Passive dynamic quadrupedal walking," in Proc. of International Conference on Robotics and Automation (ICRA1997), vol. 1, pp. 34-39, 1997.

[21] C.D. Remy, M. Hutter, and R. Siegwart, "Passive dynamic walking with quadrupeds - Extensions towards 3D," in Proc. of 2010 IEEE International Conference on Robotics and Automation (ICRA2010), pp. 5231-5236, May 2010.

[22] K. Nakatani, Y. Sugimoto, and K. Osuka, "Demonstration and analysis of quadrupedal passive dynamic walking," Advanced Robotics, vol. 23, no. 5, pp. 483-501, 2009.

[23] Y. Sugimoto, H. Yoshioka, and K. Osuka, "Development of super-multi-legged passive dynamic walking robot," in Proc. of SICE Annual Conference 2011 (SICE2011), pp. 576-579, 2011.

[24] A. Goswami, B. Espiau, and A. Keramane, "Limit cycles in a passive compass gait biped and passivity-mimicking control laws," J. of Autonomous Robots, vol. 4, no. 3, pp. 273-286, 1997.

[25] F. Asano and M. Yamakita, "Virtual gravity and coupling control for robotic gait synthesis," IEEE Transactions on Systems, Man, and Cybernetics - Part A: Systems and Humans, vol. 31, no. 6, pp. 737-745, 2001.

[26] Y. Sugimoto and K. Osuka, "Walking control of quasi-passive-dynamic-walking robot "Quartet III" based on delayed feedback control," in Proc. of 5th International Conference on Climbing and Walking Robots (CLAWAR2002), pp. 123-130, 2002. 